\title{
SOZIALVERSICHERUNG IN ÄGYPTEN
}

\author{
Von GÜNTER WIEDENSOHLER
}

Seit Entstehung der Republik Ägypten im Jahre 1952 arbeitete die Regierung beharrlich an der Verwirklichung eines ihrer wichtigsten gesellschaftspolitischen Ziele, dem Aufbau einer modernen Sozialversicherung (at-ta'mînât al-idjtimâ‘̂yya). Ein Durchgangsstadium auf dem Wege dorthin war die allgemeine Versicherungsund Versorgungskasse für Arbeitnehmer ${ }^{1}$, eine 1955 gegründete rechtsfähige öffentliche Anstalt, die vor allem das mit dem Tode oder der Erwerbsunfähigkeit eines Mitglieds verbundene finanzielle Risiko verringerte. Daneben bestand ein umfangreiches System betrieblicher Sparfonds, Gruppenversicherungen und Pensionseinrichtungen, deren Begünstigte von der allgemeinen Versicherungspflicht befreit waren. Durch die Einführung einer gesetzlichen Arbeitsunfall-Versicherung im Jahre 1958 wurden die Sozialleistungen des Staates wesentlich verbessert ${ }^{2}$. Im übrigen war der Schutz Erwerbstätiger - z. B. die ärztliche Betreuung und die Zahlung von Dienstabfindungen - weitgehend arbeitsrechtlich geregelt ${ }^{3}$. Wegen ihrer Gefährdung durch eine mögliche Insolvenz des Arbeitgebers wurden diese Maßnahmen jedoch ebenso wie das private Versorgungssystem nach und nach durch staatliche Versicherungen ersetzt ${ }^{4}$.

Das geltende ägyptische Sozialversicherungsgesetz (SVG) vom 21.3.19645 umfaßt die Rentenversicherung (Alters-, Invaliden- und Hinterbliebenenrente), die Versicherung gegen Arbeitsunfälle und Berufskrankheiten, die Krankenversicherung und die Arbeitslosenversicherung. Dabei handelt es sich um Zwangsversicherungen, denen alle Erwerbstätigen mit Ausnahme folgender Berufsgruppen angehören: Offentlich Bedienstete - für die besondere Versorgungseinrichtungen bestehen, landwirtschaftliche Arbeitskräfte - soweit nicht abweichend geregelt, freiberuflich Tätige, selbständige Gewerbetreibende, Heimarbeiter und Hauspersonal ( $\$ 2$ SVG). Versicherungsträger ist die dem Arbeitsministerium unterstehende, mit eigener Rechtspersönlichkeit ausgestattete Allgemeine Sozialversicherungs-Anstalt (ASA) ${ }^{6}$, deren Aufgaben weitgehend von fünf Regional- und 36 Bezirkszweigstellen wahrgenommen werden. Die ASA-Beamten haben das Recht, Arbeitsplätze zu besichtigen und alle erforderlichen Maßnahmen zur Abwicklung der Versicherung zu treffen ${ }^{7}$. Bei Betrieben mit mehr als 50 Arbeitnehmern ist einer von ihnen Obmann der ASA.

Der von der ASA verwaltete Fonds wird vor allem aus den Arbeitgeber- und Arbeitnehmerbeiträgen finanziert, die aus bestimmten Prozentsätzen des Arbeitsentgelts bestehen und bei jeder der vier Sozialversicherungssparten unterschiedlich hoch sind. Ebenso wie im deutschen System hat der Arbeitgeber auch die Beiträge

1 Qânûn raqm 419 li-sanat 1955 bi-inshâ' sundûq li-'t-ta'mîn wa-âkhar li-'l-iddikhảr, al-Djarîda 'r-Rasmiya, raqm 67 li-sanat 1955.

2 Qânûn raqm 202 li-sanat 1958 bi-sha'n at-ta'mîn wa-'t-ta'wîd 'an isâbât al-'amal, alDjarîda 'r-Rasmîya raqm 40 li-sanat 1958.

3 Vgl. Qânûn raqm 41 li-sanat 1944 bi-sha'n 'aqd al-'amal al-fardî, al-Waqâ'i' al-Misrîya, raqm 60 li-sanat 1944.

4 Vgl. Gerhard Fulda: Die Entwicklung des ägyptischen Sozialversicherungsrechts, Hamburg 1969, S. 44 ff.; Ahmad Ibrâhîm Hasan: Nahs'at at-ta'mînât al-idjtimâ'îya wa-tatawwuruhâ, Madjallat al-'Amal 19/1964, S. $44 \mathrm{ff}$.

5 Qânûn raqm 63 li-sanat 1964, al-Djarîda 'r-Rasmîya, raqm 67 li-sanat 1964.

6 al-Hai'a 'l-âmma li-'t-ta'mînăt al-idjtimâ'îya.

7 Vgl. im einzelnen Mahmûd Rashâd al-Haddâd: Kaifa tata'âmal ma'a hai'at at-ta'mînât al-idjtimå'îya, Mưassasat al-Ahrâm, al-Qâhira 1970, S. 5 ff., 33 ff. 
der bei ihm Beschäftigten abzuführen. Im Falle einer Deckungslücke besteht eine Zuschußpflicht des Staates. Von den Zahlungen an die ASA sind nur solche Privatunternehmen befreit, die ihren Belegschaftsangehörigen einen mindestens gleichwertigen, tarifvertraglich gesicherten Schutz gewähren. Die im Anhang zum SVG aufgeführten Versicherungsleistungen richten sich nach dem Alter des Versicherten, seinen durchschnittlichen Arbeitseinkünften, der Dauer seiner Berufstätigkeit und der Gesamthöhe der von seinem Verdienst einbehaltenen Beiträge. Die monatliche Mindestrente beträgt 360 Piaster, die Höchstrente 100 ägyptische Pfund. Ein gleichzeitiger Bezug mehrerer Renten ist ebenso wie eine Kapitalabfindung nur ausnahmsweise möglich ( $\$ 93 \mathrm{SVG}$ ).

\section{Rentenversicherung}

Die in den $\iint 75-90$ SVG geregelte Alters-, Invaliden- und Hinterbliebenenversicherung (ta'mîn ash-shaikhûkha wa-'l-'adjz wa-'l-wafât) wurde durch Gesetz Nr. 143/1961 erstmals zu einer Versicherungskasse bei der ASA zusammengefaßt. Wie schon aus der Beitragshöhe ersichtlich - Arbeitgeberanteil 14 Prozent und Arbeitnehmeranteil 8 Prozent - ist sie der bedeutendste Sozialversicherungszweig. Ihr sozialpolitischer Rang ergibt sich aus der Nationalen Charta, dem politischideologischen Manifest der VAR, in dem es heißt: „Die Versicherung gegen Alter und Krankheit muß ausgebaut werden, damit sie einen wirksamen Schutz für alle Mitbürger darstellt, die ihre Aufgabe im nationalen Kampf erfüllt haben. Alte und Kranke haben ein selbstverständliches Recht auf Ruhe und soziale Sicherheit." Die Wirtschaftsentwicklung des Landes wird hiernach als nationaler Kampf verstanden und die Sozialversicherung als Ausgleich der in diesem Kampf erlittenen Opfer und Entbehrungen.

Eine Altersrente von maximal 75 Prozent erhält, wer das 60. Lebensjahr vollendet hat und mindestens 20 Jahre lang sozialversichert war. Das Ruhegehalt setzt sich wie folgt zusammen: 2 Prozent des monatlichen Durchschnittseinkommens für jedes Beitragsjahr und je 1 Prozent für frühere Berufsjahre, die auf die Versicherungszeit angerechnet werden. Der monatliche Durchschnittsverdienst errechnet sich aus der in den letzten zwei Jahren bezogenen Gesamtvergütung des Berechtigten. Neu eingeführt wurde die Regelung, daß Versicherte - unter entsprechender Herabsetzung der Rentenbeträge - schon mit Erreichen des 50. Lebensjahres (Frauen mit dem 45. Lebensjahr) in den Ruhestand treten können. In die Versicherungsdauer werden einbezogen: die Zeit einer früheren Tätigkeit im öffentlichen Dienst, die Dauer der Mitgliedschaft in der ehemaligen öffentlichen Versicherungs- und Versorgungskasse (sundûq at-ta'mîn wa-âkhar li-'l-iddikhâr) ${ }^{10}$ sowie die Berufszeit, für die nach der früheren Gesetzgebung11 ein Anspruch auf eine arbeitsrechtliche Abfindung (mukâfa'at nihâyat al-khidma) entstanden war. Sind die Voraussetzungen für den Bezug einer Rente nicht erfüllt, weil der 60jährige Arbeitnehmer weniger als 20 Jahre versichert war, so erhält er eine Altersabfindung (ta'wîd ash-shaikhûkha) von 15 Prozent seines durchschnittlichen Jahresverdienstes, wie er sich aus den letzten 24 Monaten vor Eintritt in den Ruhestand ergibt.

8 Anwar al-'Amrûsî: Qânûn at-ta'mînât al-idjtimâ'îya, Dâr Nashr ath-Thaqâfa, al-Qâhira 1966, S. 65 ff.

9 al-Mîthâq al-watanî, Maslahat al-Isti'lâmât, al-Qâhira 1962, S. 86.

10 Qânûn raqm 419 li-sanat 1955 bi-inshâ' sundâq li-'t-ta'mîn wa-âkhar li-'l-iddikhâr, al-Djarîda 'r-Rasmíya, raqm 67 li-sanat 1955 .

11 Vgl. Qânûn raqm 317 li-sanat 1952 bi-sha'n 'aqd al-'amal al-fardî, al-Waqâ'i' al-Misrîya, raqm 107 li-sanat 1952 
Zur Vermeidung solcher Härten ermöglicht die erste Novelle zum SVG ${ }^{12}$ dit Ergänzung fehlender Versicherungsjahre, indem sie arbeitsfähigen Erwerbstätigen die Fortsetzung ihres Dienstverhältnisses über das 60. Lebensjahr hinaus gestattet.

Wer den Anwendungsbereich des SVG vor Erreichen der Altersgrenze endgültig verläßt, indem er z. B. in einen selbständigen Beruf überwechselt, erhält eine Abfindung, deren Höhe für jedes Beitragsjahr je nach Versicherungsdauer zwischen 11 Prozent und 15 Prozent und für jedes anrechnungsfähige frühere Berufsjahr 7,5 Prozent des durchschnittlichen Jahreseinkommens beträgt. Das gleiche gilt für verheiratete Frauen, die ihre Berufstätigkeit aus familiären Gründen vorzeitig aufgeben. Waren die Betreffenden 20 Jahre lang sozialversichert, so haben sie die Wahl zwischen einer sofort auszahlbaren Abfindung und einer Rente vom 60. Lebensjahr an ( $(81 \mathrm{SVG})^{13}$.

Welche administrativen Schwierigkeiten sich bei der Abwicklung der Sozialversicherung in einem Lande wie Ägypten ergeben können, veranschaulicht $\ 77$ Absatz 3 SVG: „Der Beweis, daß ein Versicherter das 60. Lebensjahr vollendet hat, wird durch Zeugenaussagen, amtliche Auszüge aus dem Geburtsregister oder andere öffentliche Urkunden erbracht. Ist die Beweisführung auf diese Weise nicht möglich, wird das Alter des Versicherten im Auftrage der ASA von einem Arzt geschätzt. Wenn der Betroffene das Ergebnis der Schätzung nicht anerkennt, entscheidet eine amtsärztliche Gutachterkommission. Der Kommissionsentscheid ist endgültig und unanfechtbar selbst dann, wenn sich später herausstellt, daß das tatsächliche Alter des Versicherten von dem amtlich festgesetzten abweicht."

Wenn der Versicherte während der Dauer des Arbeitsverhältnisses infolge außerberuflicher Ursachen stirbt oder dauernd erwerbsunfähig wird, erhalten er oder seine Hinterbliebenen eine 40prozentige Rente (ma'âsh al-'adjz au al-wafât), vorausgesetzt, daß für mindestens sechs aufeinanderfolgende oder zwölf unzusammenhängende Monate Beiträge gezahlt worden sind. Sofern dies für den Berechtigten günstiger ist, entsprechen seine Bezüge denjenigen der Altersrente, und zwar auf der Grundlage einer kraft Gesetzes um drei Jahre verlängerten Beitragszeit ( $₫ 83$ SVG). Rentenberechtigte Hinterbliebene sind Witwen, Kinder, Eltern und ausnahmsweise auch Geschwister, falls diese nämlich nachweisen, daß sie zu Lebzeiten des Versicherten ganz oder teilweise von ihm unterhalten worden sind. Witwen, Töchter und Schwestern verlieren die Rente im Falle ihrer Heirat. Das gleiche gilt von Abkömmlingen, sobald sie sich aus eigener Erwerbstätigkeit unterhalten können. Nach dem Tode des Rentenempfängers erhält die Witwe oder ein anderer gesetzlicher Erbe den Betrag einer Monatsrente, mindestens jedoch fünf ägyptische Pfund, zur Bestreitung der Bestattungskosten ${ }^{14}$.

In der Erkenntnis, daß die Invaliden- und Hinterbliebenenrente - vor allem bei jüngeren Versicherten - nur einen unvollkommenen Ausgleich darstellt, hat der Gesetzgeber in den $\int \mathbb{S} 87 \mathrm{f}$.SVG einen zusätzlichen Leistungsanspruch (at-ta'mîn al-idâfî) geschaffen. Der Begünstigte erhält eine Abfindungssumme, deren Höhe in einem altersabhängigen Prozentsatz des durchschnittlichen Jahresverdienstes besteht. Diese Sätze schwanken zwischen 267 Prozent bei einem 25jährigen und 20 Prozent bei einem 65jährigen Versicherten. Sie erhöhen sich um die Hälfte, wenn der Tod oder die Arbeitsunfähigkeit durch einen Berufsunfall bedingt ist.

12 Qânûn raqm 4 li-sanat 1969 bi-ta'dîl ba‘d ahkâm qânûn at-ta'mînât al-idjtimâ'îya, al-Ahrâm al-Iqtisâdî $323 / 1969$, S. $58 \mathrm{f}$.

13 Vgl. Mahmûd Rashâd al-Haddâd: at-Ta'mînât al-idjtimâ'îya, Mu'assasat al-Ahrâm, al-Qâhira 1967, S. 39 ff.

14 Vgl. Mahmûd 'Umar: al-Ma'âsh fî hâlat al-'adjz al-kâmil au al-wafât 'an ghair isâbat 'amal, Madjallat al-'Amal 34/1966, S. $64 \mathrm{ff}$. 
Diese Art sozialer Vorsorge trägt Merkmale einer privaten Lebensversicherung, da die Abfindung beim Tode des Versicherten nicht an die nach SVG begünstigten Hinterbliebenen geht, sondern an die gesetzlichen Erben oder andere Personen, die der Versicherte ausdrücklich benannt hat ${ }^{15}$.

\section{Unfallversicherung}

Die Arbeitsunfallversicherung (ta'mîn isâbât al-'amal) der $\int \mathbb{S}$ 19-47 SVG, die auch das Risiko von Berufskrankheiten (amrâd mihanîya) abdeckt, wird ausschließlich vom Arbeitgeber finanziert, und zwar in Höhe von 3 Prozent des Arbeitsentgelts. Pflichtmitglieder der Versicherung sind alle Beschäftigten in Industrie, Handel und Dienstleistungsgewerbe einschließlich der Kurzzeit- und Gelegenheitsarbeiter. Landwirtschaftliche Arbeitskräfte sind nur insoweit einbezogen, als sie spezifischen Berufskrankheiten ausgesetzt sind oder bei ihrer Arbeit mechanische Werkzeuge benutzen. Der Unfall im engeren Sinne (hâdith al-'amal), dessen Merkmale das SVG nicht abgrenzt, wird in Anlehnung an die ägyptische Rechtsprechung definiert als „Eintritt eines körperlichen Schadens (darar djusmânî) durch ein plötzliches, unerwartetes Ereignis (fi'l fudjầ̂ $\hat{\imath}$ ) äußeren Ursprungs (asl khâridjî̀)“16. Der Begriff des Körperschadens wird nicht abstrakt umgrenzt; vielmehr wird kasuistisch festgelegt, was hierunter $\mathrm{zu}$ verstehen ist: Verwundungen, Knochenbrüche, Verlust des Gedächtnisses, nervliche Störungen, entstellende Verletzungen usw. Das SVG qualifiziert ein derartiges Unglück als Arbeitsunfall, wenn es entweder durch die Arbeit verursacht (bi-sabab al-'amal) oder während ihrer Ausführung (athnâ' ta'diyatihî) eingetreten ist. Demgegenüber forderte die frühere Gesetzgebung ${ }^{17}$, daß beide Voraussetzungen - durch die Arbeit und während ihrer Ausführung kumulativ erfüllt sein mußten. Dem Arbeitsunfall expressis verbis gleichgestellt ist der Wegeunfall (isâbat at-tarîq) ${ }^{18}$, d. h. ein Unfall, den der Versicherte auf seinem direkten Wege zur und von der Arbeit erleidet.

Nach einem Unfall trägt die ASA die Kosten der Arzt- und Krankenhausbehandlung bis zur Wiederherstellung des Verletzten oder bis zur Feststellung seiner Invalidität. Wer vorübergehend an der Wiederaufnahme seiner Arbeit gehindert ist, erhält während dieser Zeit eine Unterstützung in voller Höhe des Lohnes. Bei dauernder Erwerbsunfähigkeit (al-'adjz al-kâmil al-mustadîm) oder Tod beträgt die Rente 80 Prozent des monatlichen Durchschnittslohnes, den der Versicherte im letzten Jahr bzw. bei kürzerer Versicherungsdauer in dieser Zeit bezogen hat. Im Falle teilweiser Invalidität (al-'adjz al-djuz'i 'l-mustadîm) erhält der Versicherte eine dem Grad seiner Erwerbsminderung entsprechende Rente. Ist seine Arbeitskraft um mehr als 65 Prozent beeinträchtigt, steht ihm eine Abfindung zu. Nach der zweiten Novelle zum SVG ${ }^{19}$ bezieht auch der in seiner Erwerbsfähigkeit Geminderte die Vollinvalidenrente, falls seine Behinderung zur Auflösung des bisherigen Arbeitsverhältnisses geführt hat und kein geeigneter neuer Arbeitsplatz zur Verfügung steht. Für Berufskrankheiten tritt die Versicherung auch dann noch ein, wenn die ersten Krankheitssymptome sich innerhalb eines Jahres nach Beendigung der gefahrenträchtigen Arbeit zeigen. Erklärt die ASA den Betroffenen für

15 Vgl. Anwar al-'Amrûsî: Qânûn at-ta'mînât al-idjtimâ'îya, S. 47 f.

16 'Abd al-Halîm al-Qâdî: Ta'mîn isâbât al-'amal, Dâr wa-Matâbi' ash-Sha'b, al-Qâhira 1966, S. 36 f.

17 Vgl. Qânûn raqm 202 li-sanat 1959 bi-sha'n at-ta'mîn wa-'t-ta'wîd 'an isấbât al-'amal, al-Djarîda 'r-Rasmîya, raqm 40 li-sanat 1958.

18 Vgl. 'Abd' al-Halîm al-Qâdî, a. a. O., S. 38 .

19 Qânûn raqm 40 li-sanat 1970 bi-ta'dỉl ba'd ahkâm qânûn at-ta'mînât al-jtimâ'îya, al-Ahrâm al-Iqtisâdî $357 / 1970$, S. 66. 
voll oder teilweise erwerbsfähig, so kann er diese Verfügung beim Arbeitsministerium anfechten, wo zwei bzw. im Falle unterschiedlicher Voten drei Amtsärzte mit bindender Wirkung entscheiden.

Das beschäftigende Unternehmen ist verpflichtet, am Arbeitsplatz für Erste Hilfe zu sorgen, den Unfall unverzüglich bei der ASA zu melden, den Verletzten zu einer Unfallstation zu transportieren, binnen 48 Stunden die Polizei zu benachrichtigen und die von bestimmten Berufskrankheiten bedrohten Arbeitnehmer behördlich zu melden, damit eine regelmäßige ärztliche Überwachung gewährleistet ist $^{20}$. Gesetz Nr. 202/1958 schreibt vor, welche Maßnahmen ein Arbeitgeber auf dem Gebiet der Unfallverhütung (wiqâya min isâbât al-'amal) und Arbeitshygiene $\mathrm{zu}$ treffen hat. Detaillierte Regelungen betreffen unter anderem Beleuchtung, Belüftung, Lärm- und Feuerschutz. In Unternehmen mit mehr als 100 Beschäftigten kontrolliert ein entsprechend ausgebildeter Arbeitnehmer die allgemeine Befolgung der Sicherheitsvorschriften. Betriebseigene Unfallverhütungs-Komitees erforschen die Unfallursachen und geben Empfehlungen zur Verbesserung des Arbeitsschutzes (al-amn as-sinâ $\hat{1} \hat{1})$. Der Komitee-Bericht wird der zuständigen Arbeitsbehörde vorgelegt; dort muß der Arbeitgeber zudem in monatlichen Abständen Unfallstatistiken einreichen ${ }^{21}$.

Der Staat läßt es nicht dabei bewenden, den Unfallopfern eine Rente zu gewähren, er bemüht sich überdies um ihre berufliche Rehabilitation (at-ta'hîl al-mihanî). Darunter sind laut $\ 26$ des geltenden Arbeitsgesetzes Nr. 91/195922 alle Maßnahmen $\mathrm{zu}$ verstehen, die dem Behinderten die Wiederaufnahme seiner früheren oder einer seinem Zustand entsprechenden Tätigkeit ermöglichen sollen. In besonderen Berufsförderungszentren (marâkiz at-ta'hîl al-mihanî) werden die Versehrten auf ihre Rückkehr ins Erwerbsleben vorbereitet ${ }^{23}$. Sobald der Beschädigte wieder arbeitsfähig ist, unterstützt ihn die Behörde bei der Unterbringung in eine geeignete Stellung. Zu diesem Zweck kann ihm und seiner Familie auf Staatskosten ein neuer Wohnsitz zugewiesen werden. Betriebe mit 50 und mehr Arbeitnehmern haben bis zu 2 Prozent ihrer Belegschaftsstärke die ihnen von der Arbeitsvermittlung vorgeschlagenen Versehrten einzustellen, wobei Kriegsinvaliden den Vorrang haben. Jedes Unternehmen muß der zuständigen Arbeitsbehörde mitteilen, wieviele Versehrte es beschäftigt, welche Tätigkeiten sie ausführen und wie sie entlohnt werden ${ }^{24}$.

\section{Krankenversicherung}

Vor Inkrafttreten der $\iint 48-62$ SVG über die Krankenversicherung (at-ta'mîn as-sihhî) war der Schutz erkrankter Arbeitnehmer ausschließlich arbeitsrechtlich geregelt ${ }^{25}$. War der Kranke arbeitsunfähig, so wurde er bei teilweiser Lohnfortzahlung beurlaubt (idjâza maradîya). Weitere Leistungen des Arbeitgebers hingen von der Betriebsgröße ab. Unternehmen mit mehr als 100 Beschäftigten mußten für ambulante ärztliche Behandlung sorgen und Medikamente zur Verfügung stellen. Bei mehr als 500 Beschäftigten mußten überdies Spezialärzte bereitstehen

20 Vgl. Ahmad Ibrâhîm Hasan: al-Idjrâ'ât fî hâlat isâbât al-'amal, Madjallat al-'Amal 31/1965, S. 70 f.

21 Vgl. Muhammad Mukhtâr "Abd al-Latîf: Himâyat al-quwa 'l-‘âmila fi' 'l-Djumhûrîya 'l-arabîya 'l-muttahida, Mu'assasat Rûz al-Yûsuf, al-Qâhira 1964, S. $39 \mathrm{ff}$.

22 al-Djarîda 'r-Rasmîya, raqm 71 li-sanat 1959.

23 Vgl. 'Abd al-Hamîd Bilâl: al-'Amal fî marâkiz at-ta'hîl al-mihanî, Madjallat al-'Amal 19/1964, S. 26 ff.

$24 \mathrm{Vgl}$. Tashghîl al-‘âdjizîn fi 'l-mudjtama' al-ishtirâkî, Madjallat al-'Amal 5/1963, S. 28 f.

25 Vgl. Gerhard Fulda: Die Entwicklung des ägyptischen Sozialversicherungsrechts, S. $111 \mathrm{ff}$. 
und Operationen durchgeführt werden können. Von diesen Lasten sollen die Arbeitgeber mit Einführung der gesetzlichen Krankenversicherung weitgehend befreit werden.

Die Beitragssätze betragen für Arbeitgeber 4 Prozent und für Arbeitnehmer 1 Prozent des Monatsverdienstes. Darüber hinaus muß der Versicherte sich an den Kosten einer ambulanten Behandlung mit drei und an den Kosten eines ärztlichen Hausbesuches mit 20 Piastern beteiligen. Bei größeren Unternehmen kann der Arbeitgeberanteil bis auf 1 Prozent ermäßigt werden, wenn das Unternehmen selbst die im Rahmen der Krankenversicherung zu erbringenden Leistungen übernimmt. Die versicherten Risiken sind Krankheit und Mutterschaft, und zwar ausschließlich des Versicherten; eine Familienhilfe ist der ägyptischen Krankenversicherung demnach unbekannt. Was das Gesetz unter Krankheit versteht, wird nicht näher definiert; lediglich Berufskrankheiten und Arbeitsunfälle sind ausgeschlossen. Die Leistungspflicht der Versicherung setzt voraus, daß für mindestens drei aufeinanderfolgende oder sonst sechs Monate, davon die letzten zwei zusammenhängend, Beiträge gezahlt worden sind. Arbeitnehmer in Betrieben mit mehr als 100 Beschäftigten genießen dagegen bereits Versicherungsschutz, sobald sie ASA-Mitglieder sind, eine in der Fachliteratur ${ }^{26}$ als sachlich ungerechtfertigt beanstandete Differenzierung.

Wer nachweislich krank ist, erhält pro Jahr während der ersten drei Monate seiner Arbeitsunfähigkeit ein Tagegeld von 75 Prozent und während der folgenden drei Monate von 85 Prozent des Lohnes. Das Tagegeld darf nicht niedriger sein als der gesetzlich garantierte Mindestlohn, und es ist frei von Steuern jeder Art sowie von Sozialversicherungsabgaben. Auf die Versicherungszeit wird die Dauer der Erkrankung gleichwohl voll angerechnet. Arbeitnehmern, die an Tuberkulose, Lepra, Geisteskrankheit oder anderen chronischen Erkrankungen leiden, wird der volle Lohn so lange fortgezahlt, bis sie die Arbeit wiederaufnehmen können oder ihre dauernde Erwerbsunfähigkeit festgestellt ist. Gegen die Nichtgewährung von Versicherungsleistungen kann der Betroffene ebenso wie im Falle der Unfallversicherung beim Arbeitsministerium Einspruch erheben.

Berufstätige Frauen erhalten einen Entbindungsurlaub von 50 Tagen und dürfen 40 Tage nach der Niederkunft nicht beschäftigt werden. In dieser Zeit bezieht die Frau ein Tagegeld von 75 Prozent ihres Lohnes, vorausgesetzt, daß sie bereits sechs aufeinanderfolgende Monate im Dienste desselben Arbeitgebers tätig war. Die Klausel wird als sozial ungerecht empfunden, da sie bewirken kann, daß eine Arbeitnehmerin, die jahrelang der Sozialversicherung angehört, dennoch bei einer Geburt leer ausgeht. Ein Vorschlag ${ }^{27}$ geht deshalb dahin, zumindest den staatlichen Wirtschaftssektor als einen und denselben Arbeitgeber im Sinne der Vorschrift zu betrachten, so daß eine berufstätige Frau die der Entbindung voraufgehenden sechs Monate bei verschiedenen Unternehmen ableisten kann. Heftigen Widerspruch hat die in $\$ 176$ des neuen Arbeitsgesetz-Entwurfs vorgesehene Verkürzung des Entbindungsurlaubs von 50 auf 30 Tage und seine Beschränkung auf drei Geburten hervorgerufen. Das Motiv des Gesetzgebers ist offensichtlich die zunehmend erkannte Notwendigkeit einer Geburtenkontrolle. Dieses Ziel dürfe man, so heißt es, aber nicht auf Kosten der Gesundheit berufstätiger Frauen erreichen

26 Mahmûd Rashâd al-Haddâd: at-Ta'mînât al-idjtimâ'îya, S. 58.

27 Mahmûd Rashâd al-Haddâd, a. a. O., S. 58. 
wollen. Nicht derart einschneidende Sanktionen seien erfolgversprechend, sondern allein eine bessere Aufklärung und gründlichere Ưberzeugung ${ }^{28}$.

Die Vorschriften über die Krankenversicherung sind nicht bereits mit Inkrafttreten des SVG zur Anwendung gelangt. Um eine möglichst reibungslose Durchführung zu gewährleisten, wahrscheinlich aber auch aus finanziellen Gründen, sollte die Krankenversicherung in den einzelnen Gouvernoraten sukzessive eingeführt werden, und zwar dergestalt, daß sie nach Ablauf von drei Jahren das ganze Staatsgebiet erfaßte ${ }^{29}$. Eingeweihte bezweifelten jedoch von vornherein $\mathrm{zu}$ Recht, daß sich dieses Ziel in der vorgesehenen Frist verwirklichen lasse. Durch Präsidialverordnung Nr.3298/196430 wurde für die Einführung der Krankenversicherung das Gouvernorat Alexandria und nachfolgend Kairo bestimmt. Dieselbe Verordnung übertrug den gesamten Bereich der medizinischen Betreuung (al-'ilâdj at-tibbî) auf die kurze Zeit vorher gegründete Allgemeine Krankenversicherungsanstalt für Angehörige des öffentlichen Dienstes ${ }^{31}$, wohingegen die finanzielle Unterstützung Erkrankter (al-ma'ûna 'l-mâlîya) im Aufgabenkreis der ASA verblieben ist. Einen entsprechenden Teil der nach wie vor von ihr eingezogenen Beiträge leitet die ASA seither an den neuen Versicherungsträger weiter.

\section{Arbeitslosenversicherung}

Wie die Krankenversicherung wurde auch die Arbeitslosenversicherung (at-ta'mîn didda 'l-batâla) erst durch das geltende SVG (\$S 63-74) eingeführt. Vorher hatte die Regierung vergeblich versucht, der Erwerbslosigkeit durch wirtschaftspolitische Maßnahmen Herr zu werden. Der Versicherungsschutz ${ }^{32}$ ist indessen nur begrenzt, da die weit verbreitete Unterbeschäftigung nicht erfaßt wird. Das gleiche gilt für Saison- und Gelegenheitsarbeiter im weitesten Sinne. Ebensowenig sind über 60 Jahre alte Arbeitnehmer sowie Familienangehörige und Privatbedienstete des Arbeitgebers in die Versicherungspflicht einbezogen.

Der Versicherungsfonds wird gespeist aus den Beiträgen der Arbeitgeber von 2 Prozent sowie der Arbeitnehmer und des Staates in Höhe von je 1 Prozent der Lohnsummen. Die Arbeitslosenversicherung ist damit der einzige Sozialversicherungszweig, an dessen laufender Finanzierung sich auch der Staat beteiligt. Der Versicherte erhält vom achten Tag der Arbeitslosigkeit an eine 50prozentige Unterstützung (ta'wîd al-batâla), sofern mindestens zwölf Monate lang, davon die letzten sechs kontinuierlich, Beiträge für ihn gezahlt worden sind. Die Unterstützungsdauer beträgt 16 Wochen, im Falle von 36 kontinuierlich entrichteten Monatsbeiträgen 28 Wochen $^{33}$. Die Zahlung der Unterstützung setzt voraus, daß der Versicherte arbeitsfähig und arbeitswillig ist, daß sein Name in der Arbeitslosenliste geführt wird und daß er das Arbeitsamt (maktab al-quwa 'l-âmila) zu den jeweils festgesetzten Zeiten aufsucht. Der Anspruch entfällt, wenn der Versicherte für eigene Rechnung arbeitet, ein geeignetes Stellenangebot ausschlägt, eine vom Arbeitsamt verfügte Ausbildung ablehnt, seine bisherige Beschäftigung auf-

$28 \mathrm{Vgl}$. Günter Wiedensohler: Zum Entwurf eines neuen ägyptischen Arbeitsgesetzes, Orient (Hamburg), 1/1970, S. 13.

29 Vgl. \$ 1 des Einführungsgesetzes zum SVG.

30 al-Djarîda 'r-Rasmîya, raqm 261 li-sanat 1964.

31 Wörtlich: Allgemeine Krankenversicherungsanstalt für die Bediensteten der Regierung, der Kommunalbehörden, öffentlichen Institutionen und staatlichen Wirtschaftsverbände.

32 Vgl. 'Abd al-Halîm al-Qâdî: at-Ta'mîn didda 'l-batâla, Dâr wa-Matâbi' ash-Sha'b, al-Qâhira 1965, S. 25 ff.

33 Vgl. im einzelnen Muhibb ad-Dîn Muhammad Sa'd: Ba'd mashâkil at-tatbîq al-'amalî haula 't-ta'mîn didda 'l-batâla, al-Ahrâm al-Iqtisâdî 319/1968, S. 56 f. 
gegeben hat oder aus einem der gesetzlichen Kündigungsgründe entlassen worden ist. $\mathrm{Zu}$ diesen Gründen gehören gemäß $\int 76$ des Arbeitsgesetzes: die Vorlage gefälschter Zeugnisse, schuldhafte Verursachung eines erheblichen Schadens, Nichtbeachtung der Arbeitsschutzbestimmungen, ungerechtfertigtes Fernbleiben von der Arbeit, Verrat von Betriebsgeheimnissen und allgemein die Verletzung wesentlicher Vertragspflichten. Gegen die Nichtgewährung der Unterstützung kann der Betroffene das zuständige Arbeitsamt anrufen, bei dem ein Viererausschuß entscheidet, bestehend aus dem Behördenvorsteher als Vorsitzendem sowie je einem Vertreter der Versicherungsaufsicht, der Staatspartei ASU ${ }^{34}$ und der Gewerkschaft ${ }^{35}$.

An dem bereits erwähnten Entwurf eines neuen Arbeitsgesetzes wird beanstandet, daß er eine bestimmte Gruppe von Erwerbslosen über Gebühr benachteiligt. So darf gemäß $\ 62$ des Entwurfes im staatlichen Wirtschaftssektor nur eingestellt werden, wer nicht innerhalb der letzten vier Jahre durch Disziplinarurteil oder Disziplinarverfügung rechtskräftig entlassen worden ist. Gegen diese Vorschrift werden verfassungsrechtliche Bedenken geäußert, da sie, wie es heißt, einer Verurteilung zu langf ristiger Arbeitslosigkeit gleichkommt ${ }^{36}$.

Die Stellenvermittlung für Arbeitslose (tauzîf wa-takhdîm al-âtilîn) ist in den IS $11 \mathrm{ff}$. des Arbeitsgesetzes geregelt. Danach kann jeder Erwerbslose unter Angabe seines Alters und Berufes, seiner Fähigkeiten und früheren Tätigkeiten verlangen, daß er bei dem für ihn zuständigen, dem Arbeitsministerium unterstehenden Arbeitsvermittlungsbüro (maktab at-tauzîf wa-'t-takhdîm) registriert wird. Diese Ämter unterstützen die Arbeitslosen bei der Suche nach einer geeigneten Stellung. Ein gemischt besetzter Ministerialausschuß fördert die Vermittlungstätigkeit durch Planungsvorschläge für die örtliche Arbeitsbeschaffung, berufslenkende Maßnahmen (at-taudjîh al-mihanî) in Anpassung an die Arbeitsmarktlage, Sicherstellung einer gleichmäßigen Behandlung der Stellenbewerber und Richtlinien für die Umsiedlung von Beschäftigungslosen entsprechend dem regionalen Arbeitskräftebedarf. Die Arbeitgeber müssen demVermittlungsbüro in regelmäßigen Abständen melden: die Zahl der beschäftigten Arbeiter und Angestellten, ihre Tätigkeit, ihr Alter und ihre Staatsangehörigkeit, frei gewordene und neu geschaffene Stellen sowie ihre Besetzung, Arbeitslage und Beschäftigungsmöglichkeiten des Unternehmens sowie die danach zu erwartende Zunahme oder Einsparung von Arbeitsplätzen.

34 Arab Socialist Union (al-Ittihâd al-ishtirâkî 'l-'arabî);

35 Vgl. Mahmûd Rashâd al-Haddâd: Bad' istifâda min ta'min al-batâla, al-Ahrâm al-Iqtisâdí 142/1965, S. 49 f. 36 Vgl. Ahmad Nabîl al-Hilâlî: Nazra ilâ mashrû́ qânûn al-'amal al-muwahhad al-djadîd, Misr al-Mưdasira 335/1969, S. 125. 